\title{
Enfermedad ocular y auditiva asociada al vitiligo
}

Eye and ear pathology associated with vitiligo

\author{
Mirian Yolanda Casas', Paola Andrea Torres² \\ 1. Médica, residente de segundo año de Dermatología, Universidad Militar Nueva Granada, Bogotá, D.C., Colombia \\ 2. Médica dermatóloga, Universidad Militar Nueva Granada, Bogotá, D.C., Colombia
}

\section{RESUMEN}

El vitiligo es un trastorno de la pigmentación cuyo origen no es claro, secundario a la pérdida de la función de los melanocitos que podría comprometer no solo los melanocitos epidérmicos, sino también, aquellos dispuestos en otros órganos, lo cual sugiere la presencia de enfermedades concomitantes como alteraciones oculares y auditivas, las cuales se revisaran en este artículo. Se propone la necesidad de un examen visual y auditivo en el abordaje integral de los pacientes con vitiligo para descartar dicho compromiso.

PALABRAS CLAVE: vitiligo, síndrome de Vogt-Koyanagi-Harada, uveítis, glaucoma, hipoacusia.

\section{ABSTRACT}

Vitiligo is a pigmentation disorder of unclear origin secondary to the loss of function of melanocytes, which could compromise not only epidermal melanocytes but also those established in other organs suggesting the presence of concomitant diseases such as ocular and auditory disturbances. In this review article those associations are reviewed. We suggest the need for a complete visual and auditory exam within the comprehensive approach to patients with vitiligo to rule out their compromise.

KEY WORDS: Vitiligo, Vogt-Koyanagi-Harada disease, uveitis, glaucoma, hypoacusis, visual acuity.

El vitiligo es una enfermedad milenaria descrita desde 1500 a. C. y definida como una enfermedad crónica adquirida que compromete los melanocitos, dando como resultado máculas acrómicas o hipocrómicas. Sin embargo, puede asociarse con condiciones como tiroiditis de Hashimoto, diabetes mellitus de tipo I y enfermedad de Addison, entre otras enfermedades autoinmunitarias, además del compromiso de otros órganos, lo cual sugiere que es una enfermedad sistémica más que un problema puramente cutáneo $(1,2)$. Desde tiempo atrás se ha asociado también con enfermedad auditiva y ocular, esta última, hasta en 66 $\%$ de los casos (30 \% de anormalidades coroideas y $5 \%$ de iritis). Estas usualmente se olvidan en el seguimiento de los pacientes, teniendo en cuenta que la enfermedad no cursa inicialmente con pérdida de la agudeza visual, por lo que siempre debe sospecharse (3-5).

\section{Correspondencia:}

Mirian Yolanda Casas, Carrera

Email:

minchoc414@hotmail.com

Recibido: 10de julio de 2016 Aceptado: 31 de noviembre de 2016

No se reportan conflictos de interés. 


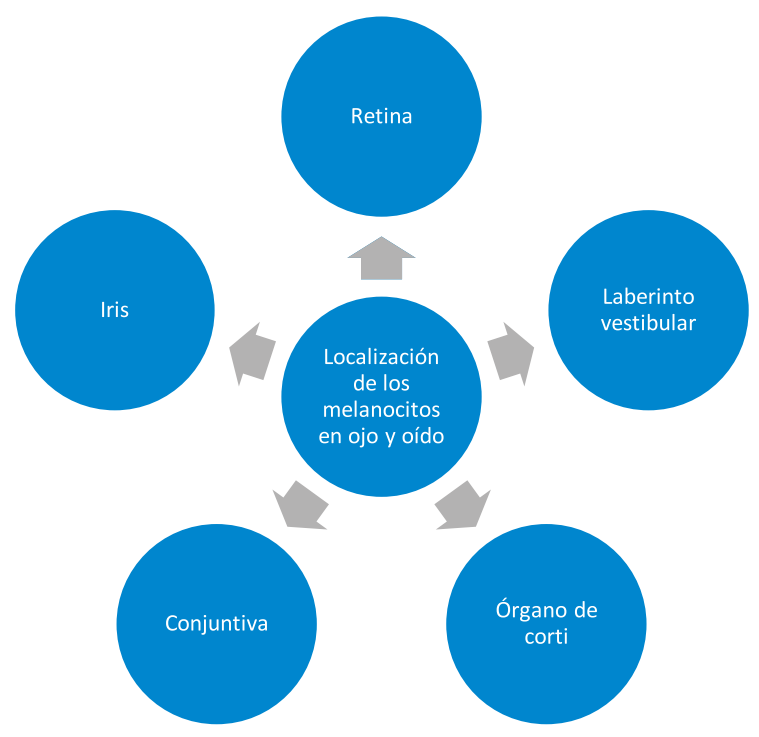

FIGURA 1. Localización de los melanocitos en ojo y oído

\section{EMBRIOLOGÍA Y MIGRACIÓN DE LOS MELANOCITOS}

Hacia la cuarta semana de la vida intrauterina, se inicia la formación del neuroectodermo y, a partir de él, se forma la cresta neural, cuyas células son las precursoras de los melanocitos. Las regiones de la cresta neural craneal y del tronco originan, entre otras células, a los melanocitos de la cabeza y del resto del cuerpo, respectivamente. Este proceso requiere la regulación de múltiples factores de transcripción, como FoxD3, Sox10, Pax3 y MITF para la regulación de su formación y, finalmente, la señalización por parte del factor de crecimiento de la familia endotelina que induce la migración continua dorso-lateral de los melanoblastos dirigida hacia sus destinos finales: la piel, el folículo piloso, la mucosa oral, las meninges, el oído interno y el ojo; en este último, en coroides, iris, retina y conjuntiva (3-5).

En el oído, los melanocitos se encuentran en el órgano de Corti, donde se localizan los receptores auditivos que, a su vez, se encuentran en la membrana basilar de la cóclea, donde se denominan células intermedias (capa intermedia de la estría vascular) y también están presentes en el laberinto vestibular (en la base de la crista ampullaris en cada canal semicircular) (6). En la cóclea, la melanina tiene un rol importante: prevenir el estrés oxidativo al limitar la formación de radicales libres $(7,8)$ (figura 1$)$.

\section{EPIDEMIOLOGÍA Y CLASIFICACIÓN DEL VITILIGO}

El vitiligo es la causa más frecuente de despigmentación de la piel, con una prevalencia estimada de 0,5 a $1 \%$, sin diferencias por sexo ni raza, con mayor afectación antes de los 20 años $(9,10)$. La forma más común de presentación es la no segmentaria (mucosa, universal, generalizada, acrofacial o mixta), caracterizada por lesiones distribuidas en un patrón simétrico, y la segmentaria, que corresponde a 5 a $16 \%$ de los casos, con un patrón en banda unilateral $(9,11,12)$.

Existen múltiples teorías para explicar la ausencia localizada de los melanocitos, entre ellas, el estrés oxidativo, la disfunción mitocondrial y mecanismos inmunológicos; ninguna brinda una explicación totalmente satisfactoria $(13,14)$.

\section{ASOCIACIÓN DE ENFERMEDAD OCULAR}

La relación de vitiligo con enfermedad ocular no es nueva y se han descrito dos síndromes clínicos en los cuales coexisten: la oftalmía simpática, que se relaciona con un antecedente de trauma intraocular, y el síndrome de Vogt-Koyanagi-Harada $(15,16)$. Estas enfermedades son de origen autoinmunitario, mediadas por linfocitos $\mathrm{T}$ con reacción contra antígenos del melanocito en la coroides, la cóclea, las meninges y la piel; hay mayor compromiso sistémico en el síndrome de Vogt-Koyanagi-Harada $(17,18)$.

En modelos animales, como el porcino Sinclair que tiene una predisposición espontánea para desarrollar vitíligo, también se ha visto la asociación con compromiso del iris $(10,19)$.

En varios estudios se ha sugerido una asociación entre la enfermedad ocular y el vitiligo, entre ellos, un estudio iraní con 72 pacientes en el cual encontraron $11 \%$ de prevalencia en pacientes de vitiligo frente a 4 $\%$ en el grupo control. Se encontró hipopigmentación del epitelio retiniano, atrofia periférica del iris, atrofia del epitelio pigmentario retiniano e hiperpigmentación de la retina (20). En otro estudio en India, se encontró enfermedad ocular específica en $16 \%$ de los pacientes frente a $5 \%$ del grupo control, como heterocromía del iris, defecto en la agudeza visual, uveítis, hipopigmentación o hiperpigmentación de la retina; también, hubo otras inespecíficas, como cataratas y conjuntivitis en el 
$25 \%$ frente al $8 \%$ en el grupo control, y cuya única sintomatología puede ser ceguera nocturna (21).

\section{ENFERMEDADES OCULARES ASOCIADAS A VITILIGO}

Glaucoma de ángulo abierto. Usualmente, se ha relacionado el glaucoma en pacientes con vitiligo al uso de corticoides. Sin embargo, en un estudio con algunas limitaciones por el tamaño de la muestra, se practicó examen oftalmológico a 42 pacientes con vitiligo sin manejo con corticoides y se encontró glaucoma de ángulo abierto en 57 \% (24), con una p significativa con factores asociados al desenlace, como edad de más de 56 años y tiempo de duración del vitiligo mayor de 13 años (22)dermatovenereologists \nand ophthalmologists to the possible association of vitiligo and ocular findings \ncharacteristic of primary open angle glaucoma (POAG.

En otro estudio, en el cual se excluyeron pacientes con hipertensión arterial, enfermedades del tejido conjuntivo o diabetes mellitus de tipo II, encontraron cambios hemodinámicos en la arteria oftálmica relacionados con eventos isquémicos determinados mediante ultrasonido Doppler a color que se correlacionaban con glaucoma de ángulo abierto sin una causa clara; por esto, recomiendan el estudio oftalmológico de rutina en los pacientes con vitiligo (23).

Alteración conjuntival y de la película lagrimal. En un estudio se incluyeron 30 pacientes con vitiligo periocular sin tratamiento previo, ni diagnóstico de otra enfermedad autoinmunitaria u ocular de base, y un grupo control de 50 pacientes sin vitiligo. Se encontró una adecuada función de la glándula lacrimal mediante la prueba de Schirmer, pero un tiempo más corto de ruptura de la película lacrimal, asociado a mayor presencia de un patrón inflamatorio y disminución de las células caliciformes (productoras de mucina de la película lacrimal), en la biopsia de la superficie ocular del grupo con vitiligo periocular comparado con el grupo control; los autores no descartaban que estos cambios fueran secundarios a enfermedades autoinmunitarias asociadas a vitiligo, no diagnosticadas (24). En otros dos estudios en los cuales evaluaron también la prueba de Schirrmer y el tiempo de ruptura de la película lacrimal, obtuvieron resultados similares, pero además, hallaron un mayor compromiso en estas pruebas en casos de vitiligo periocular y acrofacial $(25,26)$.

Uveítis. En India, en un estudio ya mencionado, encontraron uveítis en $4 \%$ de 150 pacientes con vitiligo, entre ellos, uno con panuveítis, dos con uveítis anterior y tres con uveítis intermedia, sin ningún caso en el grupo control (21).

Vitiligo coroideo. Puede ser primario o secundario. El primario se manifiesta usualmente como parches extensos de hipopigmentación coroidea, sin compromiso retiniano, en pacientes que presentan vitiligo cutáneo. El secundario se relaciona con procesos inflamatorios uveales, en enfermedades como el síndrome de VogtKoyanagi-Harad; este es subdiagnosticado y podría confundirse con uveítis multifocal (34-37).

Halo nevus coroideo. Es un nevus moteado, con un área de despigmentación alrededor, con crecimiento progresivo (27).

Iris. En dos estudios, la presencia de parches hipopigmentados, en 23 a $33 \%$ de los casos, fue el hallazgo más frecuente $(28,29)$.

Trastornos pigmentarios de la retina. Desde 1977 se ha encontrado asociación entre hipopigmentación del epitelio retiniano y atrofia de los fotorreceptores con nictalopía posiblemente secundaria, en $25 \%$ de los pacientes con vitiligo; además, es posible que exista una asociación con retinitis pigmentaria, al parecer, sin disminución del espesor de la capa de fibras nerviosas de la retina (30-33).

En el estudio histolpatológico de ratones mutados con vitiligo, también se ha visto una alteración estructural en las células fotorreceptoras, despigmentación de la cabeza del nervio óptico, infiltración subrretiniana por macrófagos y desprendimiento de la retina, cambios que se correlacionan con alteraciones en el electrorretinograma y progresión del vitiligo $(34,35)$. Estas alteraciones en los fotorreceptores podrían estar relacionadas con una mayor sensibilidad de la retina a la luz, por disminución en la cantidad de pigmento (35). Sin embargo, también podría tener alguna injerencia la raza, ya que en un estudio en pacientes con fototipos oscuros solo se encontraron algunas alteraciones pigmentarias maculares sutiles, lo cual contrasta con los hallazgos en caucásicos con vitiligo (36). La ausencia de síntomas podría explicarse por la presencia de lesiones en la periferia y no en cercanía de la mácula (28).

Trastornos electrofisiológicos retinianos. En modelos de ratón con vitiligo, se han evidenciado anormalidades en los fotorreceptores, entre ellos, apoptosis y disminución en la adhesión entre el epitelio retiniano pigmentario y la retina neural. Además, en un estudio de 21 pacientes con vitiligo, sin exposición a la fototerapia, el electrorretinograma (que permite evaluar cambios en la corriente de la retina, secundarios a un estímulo luminoso) demostró alteración de la electrofisiología de la retina, con disminución de la amplitud de las ondas a y b, con las cuales se evalúan las corrientes eléctricas de los fotorreceptores 
TABLA 1. Estudios con hallazgos oculares en vitíligo

\begin{tabular}{|c|c|c|}
\hline AUTOR & $\begin{array}{l}\text { NÚMERO DE PACIENTES CON } \\
\text { VITÍLIGO EN EL ESTUDIO/TIPO } \\
\text { DE POBLACIÓN/CONTROLES }\end{array}$ & HALLAZGOS EN PACIENTES CON VITÍLIGO \\
\hline $\begin{array}{l}\text { Albert, et al., } \\
1979\end{array}$ & 112 humanos/sin controles & $\begin{array}{l}\text { Uveítis, atrofia de la capa pigmentaria del iris, atrofia peripapilar, } \\
\text { papiledema, atrofia óptica, síntomas de ceguera nocturnos, } \\
\text { defectos de transiluminación del iris }\end{array}$ \\
\hline $\begin{array}{l}\text { Lentz, et al., } \\
1983\end{array}$ & $\begin{array}{l}30 \text { cerdos Sinclair con vitíligo } \\
\text { inducido genéticamente }\end{array}$ & Despigmentación del iris, despigmentación del fondo de ojo \\
\hline $\begin{array}{l}\text { Wagoner, et } \\
\text { al., } 1983\end{array}$ & 223 humanos & $\begin{array}{l}27 \% \text { hipopigmentación del epitelio retiniano, } 25 \% \text { refiere ceguera } \\
\text { nocturna }\end{array}$ \\
\hline $\begin{array}{l}\text { Albert, et al., } \\
1983\end{array}$ & $\begin{array}{l}223 \text { humanoscon psoriasis y } \\
\text { fototerapia }\end{array}$ & $\begin{array}{l}27 \% \text { con algún tipo de hipopigmentación del epitelio pigmentado } \\
\text { de la retina y } 25 \% \text { se queja de ceguera nocturna y o,8 \% retinitis } \\
\text { pigmentaria. }\end{array}$ \\
\hline $\begin{array}{l}\text { Nir, et al., } \\
1995\end{array}$ & $\begin{array}{l}40 \text { ratones mutados } \\
\text { C57Bl/6-mi”"/mi”” }\end{array}$ & $\begin{array}{l}\text { Degeneración de la retina ecuatorial y posterior, acumulo } \\
\text { de detritos subretinianos previos a la muerte de las células } \\
\text { fotorreceptoras }\end{array}$ \\
\hline $\begin{array}{l}\text { Tang, et al., } \\
1997\end{array}$ & $\begin{array}{l}16 \text { ratones mutados } \\
\left(\mathrm{C}_{57} \mathrm{Bl} / 6-\mathrm{mi}, ” / \mathrm{mi} " ”\right)\end{array}$ & $\begin{array}{l}\text { Alteración estructural en fotorreceptores, despigmentación de la } \\
\text { cabeza del nervio óptico, infiltración subretiniana por macrófagos } \\
\text { y desprendimiento de la retina }\end{array}$ \\
\hline $\begin{array}{l}\text { Biswas, et al., } \\
2003\end{array}$ & 100 humanos & $\begin{array}{l}23 \% \text { parches hipopigmentados del iris, } 18 \% \text { pigmentación en la } \\
\text { cámara anterior, } 9 \% \text { hipopigmentación del epitelio pigmentario de } \\
\text { la retina, } 5 \% \text { uveítis, } 11 \% \text { degeneración coriorretiniana }\end{array}$ \\
\hline $\begin{array}{l}\text { Ayotunde, et } \\
\text { al., } 2005\end{array}$ & $\begin{array}{l}17 \text { humanos de fototipo } \\
\text { oscuro }\end{array}$ & $\begin{array}{l}\text { Sin síntomas oculares, solo cambios sutiles pigmentarios } \\
\text { retinianos }\end{array}$ \\
\hline $\begin{array}{l}\text { Shoeibi, et } \\
\text { al., } 2014\end{array}$ & 21 humanos sin fototerapia & $\begin{array}{l}\text { Las ondas de respuesta estándar, de respuesta combinado y de } \\
\text { destello de cono único tenían amplitudes significativamente } \\
\text { menores que las de la población normal. }\end{array}$ \\
\hline $\begin{array}{l}\text { Bulbul } \\
\text { Baskan, et al., } \\
2006\end{array}$ & 45 humanos & $\begin{array}{l}\text { Atrofia peripapilar alrededor del nervio óptico, atrofia del epitelio } \\
\text { pigmentario, máculas hipopigmentadas focales y difusas en } \\
\text { relación con compromiso periorbitario y genital por vitíligo. }\end{array}$ \\
\hline $\begin{array}{l}\text { Guadarrama, } \\
\text { et al., } 2006\end{array}$ & $\begin{array}{l}56 \text { humanos, población } \\
\text { pediátrica }\end{array}$ & 8,9 \% hipopigmentación retiniana. \\
\hline $\begin{array}{l}\text { Gopal, et al., } \\
2007\end{array}$ & 150 humanos & $\begin{array}{l}\text { - } 16 \% \text { heterocromia del iris, defecto en la agudeza visual, uveítis, } \\
\text { hipo o hiperpigmentación de la retina; y cataratas y conjuntivitis } \\
\text { en } 25 \% \\
\text { - } 20 \% \text { hipoacusia }\end{array}$ \\
\hline $\begin{array}{l}\text { Rogošić, et } \\
\text { al., } 2010\end{array}$ & $\begin{array}{l}42 \text { pacientes con sospecha de } \\
\text { glaucoma, humanos }\end{array}$ & $56 \%$ glaucoma primario de ángulo abierto \\
\hline
\end{tabular}




\begin{tabular}{|c|c|c|}
\hline AUTOR & $\begin{array}{l}\text { NÚMERO DE PACIENTES CON } \\
\text { VITÍLIGO EN EL ESTUDIO/TIPO } \\
\text { DE POBLACIÓN/CONTROLES }\end{array}$ & HALLAZGOS EN PACIENTES CON VITÍLIGO \\
\hline $\begin{array}{l}\text { Abdollahi, et } \\
\text { al., } 2011\end{array}$ & 72 humanos & $\begin{array}{l}15,3 \% \text { hipopigmentación e hiperpigmentación retiniana, atrofia } \\
\text { periférica del iris, atrofia del epitelio pigmentario retiniano. }\end{array}$ \\
\hline $\begin{array}{l}\text { Serin, et al., } \\
2012\end{array}$ & 30 humanos & $\begin{array}{l}61,7 \text { \% tiempo de ruptura de la película lagrimal más corto, } \\
\text { presencia de patrón inflamatorio y disminución de las células } \\
\text { caliciformes }\end{array}$ \\
\hline $\begin{array}{l}\text { Örnek, et al., } \\
2013\end{array}$ & 34 humanos, pacientes sanos & $\begin{array}{l}\text { Espesor menor de la capa de fibras nerviosas de la retina en el } \\
\text { sector superior de los dos ojos, el resto de localizaciones sin } \\
\text { cambios. }\end{array}$ \\
\hline $\begin{array}{l}\text { Duplancić et } \\
\text { al, } 2013\end{array}$ & $\begin{array}{l}42 \text { pacientes con sospecha de } \\
\text { glaucoma, humanos }\end{array}$ & $\begin{array}{l}56 \% \text { cambios en el índice de pulsatilidad y resistencia de la arteria } \\
\text { oftálmica compatible con glaucoma }\end{array}$ \\
\hline $\begin{array}{l}\text { Dogan, et al., } \\
2015\end{array}$ & 30 humanos, pacientes sanos & $\begin{array}{l}\text { Menor tiempo de ruptura de la película lagrimal con fluoresceína, } \\
\text { menor resultado de test de Schirmer y mayor frecuencia de tinción } \\
\text { con fluoresceína de la córnea. }\end{array}$ \\
\hline $\begin{array}{l}\text { Güngör et al, } \\
2015\end{array}$ & 34 humanos & $\begin{array}{l}\text { Disminución en producción lagrimal, test de Schirmer y tiempo de } \\
\text { ruptura de película lagrimal menor; y un score de rosa de Bengala } \\
\text { mayor (positivo en presencia de células muertas o desvitalizadas); } \\
\text { con peores resultados de estas pruebas en vitíligo centrofacial. }\end{array}$ \\
\hline $\begin{array}{l}\text { Pai, et al., } \\
2015\end{array}$ & 75 humanos, 75 sanos & $\begin{array}{l}\text { 33,3\% hipopigmentación o atrofia del iris, } 20 \% \text { pigmentación del } \\
\text { ángulo de la cámara anterior, atrofia peripapilar, hipopigmentación } \\
\text { difusa retiniana, fondo teselado }\end{array}$ \\
\hline
\end{tabular}

(conos, bastones y células ganglionares) y las células bipolares, respectivamente (37). Además, se han encontrado alteraciones en los potenciales evocados visuales y en la electrooculografía, correlacionadas con un compromiso cutáneo más extenso y prolongado del vitiligo (37).

Síndrome de Vogt-Koyanagi-Harada. Es una enfermedad autoinmunitaria que ataca el tejido con contenido de melanina, principalmente, el ocular. Se inicia con síntomas virales (fotofobia, cefalea y fiebre), seguidos por panuveítis aguda difusa simétrica, hiperemia, edema del disco óptico y desprendimiento retininiano seroso, y en la fase de convalecencia, se produce despigmentación del tegumento y la coroides. Las complicaciones incluyen cataratas (10-42 \%), glaucoma (6-45\%), fibrosis subretiniana (8-40 \%) y membranas neovasculares (9-14 \%). Otros órganos que tienen melanina pueden afectarse, como el sistema nervioso central (pleocitosis, cefalea, meningoencefalitis), la piel (vitiligo, poliosis, alopecia) y el oído (pérdida auditiva coclear de alta frecuencia, vértigo y alteración del reflejo óculo-vestibular) (38-40).
Además, se ha encontrado una mayor asociación entre el vitiligo periocular y la enfermedad ocular, y una relación no clara con compromiso genital $(28,41)$; igualmente, los hallazgos oculares son más frecuentes entre los pacientes de 30 a 60 años de edad con vitiligo (42).

En nuestro país no existen estudios al respecto. En población de raza similar en América, solo existe un estudio de 56 niños mexicanos, en el cual se encontró hipopigmentación retiniana en 8,9 \% de los casos, principalmente en el polo posterior, y otras alteraciones que no se creen relacionadas con el vitiligo, como ametropía (19,6\%), reacción folicular conjuntival inespecífica (7,14 $\%)$, meibomitis (5,3\%) y conjuntivitis (3,5\%) (43).

\section{ASOCIACIÓN DE ENFERMEDAD AUDITIVA}

La hipoacusia es una característica en condiciones como el piebaldismo y la enfermedad de 
Vogt-Koyanagi-Harada, lo cual sugiere una asociación entre los melanocitos y la audición (44-45). Se cree que en el vitiligo, simultáneamente con la pérdida de los melanocitos epidérmicos, se pierden células del oído interno que contienen melanina, lo cual abole su función preventiva y lo predispone a ser afectado por factores ambientales dañinos y, finalmente, lleva a pérdida de la audición neurosensorial (46).

Tosti, et al. (44), Sharma, et al. (6), y Orecchia, et al. (47) reportaron hipoacusia principalmente neurosensorial en 8 a $18,89 \%$ de los pacientes con vitiligo, con una diferencia estadísticamente significativa $(\mathrm{p}<0,01)$ con respecto a personas sanas; se excluyeron pacientes con otras enfermedades autoinmunitarias $u$ concomitantes. No hubo diferencias según edad, sexo ni tratamiento previo para vitiligo, incluidos los psoralenos $(6,44,47)$.

En otro estudio, los umbrales de audición tanto en la audiometría de tonos puros como en la de tonos de alta frecuencia (estos últimos percibidos en la estría vascularis) fueron mayores en los pacientes con vitíligo; también hubo un umbral más alto en los tonos puros entre pacientes con vitíligo activo con respecto a los que presentaban enfermedad estable (48). Además, en los pacientes con vitíligo las amplitudes de alta frecuencia (4.000 y $8.000 \mathrm{~Hz}$ ) eran significativamente más bajas durante emisiones otoacústicas evocadas por estímulos transitorios y había alteraciones en los potenciales de sumay en potenciales de acción por electrococleografía lo que sugiere alteraciones preganglionares (sugiriendo patología coclear) $(48,49)$.

Se sugiere que la asociación entre el vitiligo y las enfermedades oculares y auditivas se debe a la agresión contra los melanocitos presentes en estos órganos sensitivos (pese a ser heterogéneos con respecto a los epidérmicos) por parte de los diferentes mecanismos propuestos en la fisiopatología del vitíligo, como: los causados por los linfocitos $\mathrm{T}$, principalmente CD8+ y Th17, TNF- $\alpha$, IFN- $\gamma$, IL-17, y los anticuerpos contra antígenos del citoplasma y la membrana del melanocito. Esta actúa como moduladora de la transducción del estímulo auditivo en el oído interno además de participar en la protección del daño oxidativo celular ocular y vestibular, entre otros. Además, podrían

TABLA 1. Estudios con hallazgos oculares en vitíligo

\begin{tabular}{|c|c|c|}
\hline AUTOR & $\begin{array}{l}\text { NÚMERO DE PACIENTES } \\
\text { CON VITÍLIGO/ } \\
\text { POBLACIÓN }\end{array}$ & HALLAZGOS EN PACIENTES CON VITÍLIGO \\
\hline Tosti, et al., 1987 & 50 humanos & $16 \%$ hipoacusia \\
\hline $\begin{array}{l}\text { Orecchia, et al., } \\
1989\end{array}$ & 50 humanos & $8 \%$ pérdida auditiva de tonos altos $(4.000 \mathrm{~Hz})$ \\
\hline Özüer, et al., 1998 & 50 humanos sanos & $4 \%$ pérdida auditiva de tonos altos $(4.000$ y $8.000 \mathrm{~Hz})$ \\
\hline Hong, et al., 2009 & 89 humanos & $\begin{array}{l}\text { Umbrales de tonos más bajos a } 1.000,4.000,6.000 \text { y } 8.000 \mathrm{~Hz} \text {, } \\
\text { aumento en los potenciales de suma y en la amplitud de los } \\
\text { potenciales de acción por electrococleografía }\end{array}$ \\
\hline $\begin{array}{l}\text { Ruiz-Allec, et al., } \\
2009\end{array}$ & $\begin{array}{l}10 \text { humanos con sindrome } \\
\text { de Vogt-Koyanagi-Harada } \\
\text { y síntomas vestibulares }\end{array}$ & $\begin{array}{l}\text { Alteración de la aferencia vestibular y ocular, vértigo paroxístico } \\
\text { benigno y alteración en la audiometría tonal }\end{array}$ \\
\hline Aslan, et al., 2010 & 22 humanos & $\begin{array}{l}\text { 36,4 \% pérdida de audición en tonos puros en la audiometría y } \\
\text { disminución de los potenciales evocados transitorios otoacústicas } \\
\text { a } 4 \mathrm{kHz} \text {. }\end{array}$ \\
\hline Akay, et al., 2010 & 890 humanos & $37,7 \%$ pérdida auditiva entre 4.000 y $8.000 \mathrm{~Hz}$ \\
\hline $\begin{array}{l}\text { Al-Mutairi y Al- } \\
\text { Sebeih, } 2011\end{array}$ & $\begin{array}{l}197 \text { humanos }>40 \text { años/ } \\
\text { sanos }\end{array}$ & $22 \%$ hipoacusia \\
\hline
\end{tabular}


estar relacionadas vías inmunológicas afines, como la vía común de participación de Th17 en vitíligo, y en la disfunción lagrimal en el síndromede ojo seco (25,35,50-53).

En conclusión, no hay suficientes estudios sobre la enfermedad ocular ni la auditiva en el vitíligo. Sin embargo, se ha encontrado una mayor frecuencia de uveítis y de alteraciones retinianas, y una relación con el glaucoma de ángulo abierto y la hipoacusia neurosensorial. Por esto, los pacientes con vitiligo deberían someterse a un examen oftalmológico, que incluya agudeza visual, tonometría y prueba de Schirmer, y a una valoración auditiva de rutina. Además, deben desarrollarse estudios sobre estos aspectos en la población colombiana, hasta el momento inexistentes, ya que los hallazgos parecen variar según la raza $(22,23,53,54)$.

\section{REFERENCIAS}

1. Tarlé RG, Nascimento LM, Mira MT, Castro CCS. Vitiligo-Part 1. An Bras Dermatol. 2014;89:461-70.

2. Mahdi P, Rouzbahani M, Amali A, Rezaii Khiabanlu S, Kamali M. Audiological manifestations in vitiligo patients. Iran J Otorhinolaryngol. 2012;24:35-40.

3. Sommer L. Generation of melanocytes from neural crest cells. Pigment Cell Melanoma Res. 2011;24:411-21.

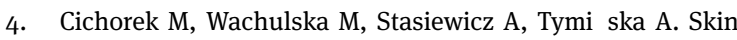
melanocytes: Biology and development. Adv Dermatol Allergol. 2013;1:30-41.

5. Hu D-N, McCormick SA, Seedor JA, Ritterband DC, Shah MK. Isolation, purification and cultivation of conjunctival melanocytes. Exp Eye Res. 2007;84:655-62.

6. Sharma L, Bhawan R, Jain R. Hypoacusis in vitiligo. Indian J Dermatol Venereol Leprol. 2004;70:162-4.

7. Roberts DS, Linthicum FH. Distribution of melanocytes in the human cochlea, Otol Neurotol. 2015;36:e99-100.

8. Barozzi S, Ginocchio D, Socci M, Alpini D, Cesarani A. Audiovestibular disorders as autoimmune reaction in patients with melanoma. Med Hypotheses. 2015;85:336-8.

9. Ezzedine K, Eleftheriadou V, Whitton M, van Geel N. Vitiligo. Lancet. 2015;386:74-84.

10. Essien KI, Harris JE. Animal models of vitiligo: Matching the model to the question. Dermatol Sin. 2014;32:240-7.

11. Taieb A, Alomar A, Böhm M, Dell'Anna ML, De Pase A, Eleftheriadou V, et al. Guidelines for the management of vitiligo: the European Dermatology Forum consensus. Br J Dermatol. 2013;168:5-19.

12. Faria AR, Tarlé RG, Dellatorre G, Mira MT, de Castro CCS. Vitiligo-Part 2-classification, histopathology and treatment. An Bras Dermatol. 2014;89:784-90.

13. Bellei B, Pitisci A, Ottaviani M, Ludovici M, Cota C, Luzi F, et al. Vitiligo: A possible model of degenerative diseases. PloS One. 2013;8:e59782.

14. Laddha NC, Dwivedi M, Mansuri MS, Gani AR, Ansarullah M, Ramachandran $\mathrm{AV}$, et al. Vitiligo: Interplay between oxidative stress and immune system. Exp Dermatol. 2013;22:245-50.
15. Cowan CL Jr, Halder RM, Grimes PE, Chakrabarti SG, Kenney JA Jr. Ocular disturbances in vitiligo. J Am Acad Dermatol. 1986;15:17-24.

16. Tsuruta D, Hamada T, Teramae H, Mito H, Ishii M. Inflammatory vitiligo in Vogt-Koyanagi-Harada disease. J Am Acad Dermatol. 2001;44:129-31.

17. Chan EW, Sanjay S, Chang BCM. Headache, red eyes, blurred vision and hearing loss. Can Med Assoc J. 2010;182:1205-9.

18. Arévalo JF, García R, Al-Dhibi H, Sánchez J, Suárez-Tata L. Update on sympathetic ophthalmia. Middle East Afr J Ophthalmol. 2012;19:13.

19. Lentz KJ, Burns RP, Loeffler K, Feeney-Burns L, Berkelhammer J, Hook RR. Uveitis caused by cytotoxic immune response to cutaneous malignant melanoma in swine: Destruction of uveal melanocytes during tumor regression. Invest Ophthalmol Vis Sci. 1983;24:1063-9.

20. 20. Abdollahi A, Hallaji Z, Ghiasi M, Afzal AZ. Ocular findings in patients with vitiligo: A case- control study. Tehran Univ Med J. 2011;68:656.

21. Gopal KV, Rama Rao GR, Kumar YH, Appa Rao MV, Vasudev P; Srikant. Vitiligo: A part of a systemic autoimmune process. Indian J Dermatol Venereol Leprol. 2007;73:162-5.

22. 22. Rogosi V, Boji L, Puizina-Ivi $\square$ N, Vanjaka-Rogosi $\square$ L, Titli $\square$ M, Kovacevi $\square$, et al. Vitiligo and glaucoma - an association or a coincidence? A pilot study. Acta Dermatovenerol Croat. 2010;18:21-6.

23. Duplanci $\square$ D, Rogosi $\square$ V, Puizina-Ivi $\square$ N, Rogosi $\square$ LV, Luksi $\square$ B, Kovaci V, et al. Prognostic value of ophthalmic artery color Doppler sonography for progression to glaucoma in vitiligo patients. Acta Med Croatica. 2013;67:47-52.

24. Serin D, Buttanri IB, Parlak AH, Boran C, Tirak E. Impression cytology of the ocular surface and tear function in patients with periocular vitiligo. Eur J Ophthalmol. 2012;22:734-8.

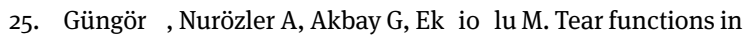
patients with vitiligo. Int J Dermatol. 2015;54:e466-8.

26. 26. Dogan AS, Atacan D, Durmazlar SP, Acar M, Gurdal C. Evaluation of dry eye findings in patients with vitiligo. Pak J Med Sci. 2015;31:587-91.

27. Fournier GA, Albert DM, Wagoner MD. Choroidal halo nevus occurring in a patient with vitiligo. Surv Ophthalmol. 1984;28:671-2.

28. Pai SG. Ocular manifestations of vitiligo: Evaluation and study of any possible association. Online J Health Allied Sci. 2016;14:1-3.

29. Biswas G, Barbhuiya JN, Biswas MC, Nazarul Islam MD, Dutta S. Clinical pattern of ocular manifestations in vitiligo. J Indian Med Assoc. 2003;101:478-80.

30. 30. Albert DM, Nordlund JJ, Lerner AB. Ocular Abnormalities Occurring with Vitiligo. Ophthalmology. 1979;86:1145-58.

31. Albert DM, Wagoner MD, Pruett RC, Nordlund JJ, Lerner AB. Vitiligo and disorders of the retinal pigment epithelium. $\mathrm{Br} \mathrm{J}$ Ophthalmol. 1983;67:153-6.

32. 32. Wagoner MD, Albert DM, Lerner AB, Kirkwood J, Forget BM, Nordlund JJ. New observations on vitiligo and ocular disease. Am J Ophthalmol. 1983;96:16-26.

33. 33. Örnek N, Onaran Z, Koçak M, Örnek K. Retinal nerve fiber layer thickness in vitiligo patients. J Res Med Sci. 2013;18:405-7.

34. Tang M, Pawlyk BS, Kosaras B, Berson EL, Sidman RL. ERG abnormalities in relation to histopathologic findings in vitiligo mutant mice. Exp Eye Res. 1997;65:215-22. 
35. Nir I, Ransom N, Smith SB. Ultrastructural features of retinal dystrophy in mutant vitiligo mice. Exp Eye Res. 1995;61:363-77.

36. Ayotunde A, Olakunle G. Ophthalmic assessment in black patients with vitiligo. J Natl Med Assoc. 2005;97:286-7.

37. Shoeibi N, Taheri A, Nikandish M, Omidtabrizi A, Khosravi N. Electrophysiologic evaluation of retinal function in patients with psoriasis and vitiligo. Doc Ophthalmol. 2014;128:131-6.

38. 38. Sakata VM, da Silva FT, Hirata CE, de Carvalho JF, Yamamoto JH. Diagnosis and classification of Vogt-Koyanagi-Harada disease. Diagn Criteria Autoimmune Dis. 2014;13:550-5.

39. 39. Greco A, Fusconi M, Gallo A, Turchetta R, Marinelli $\mathrm{C}$, Macri GF, et al. Vogt-Koyanagi-Haradasyndrome. Autoimmun Rev. 2013;12:1033-8.

40. Ruiz-Allec LD, Peñaloza-López YR, Ocaña-Plante N del R, Valdivia-Muñoz MB, Martínez-Castro F. Vestibular findings in patientswithVogt-Koyanagi-Haradasyndrome. Acta Otorrinolaringol. 2009;60:305-10.

41. Bulbul Baskan E, Baykara M, Ercan I, Tunali S, Yucel A. Vitiligo and ocular findings: A study on possible associations. J Eur Acad Dermatol Venereol. 2006;20:829-33.

42. Shankar DSK, Shashikala K, Madala R. Clinical patterns of vitiligo and its associated co morbidities: A prospective controlled cross-sectional study in South India. Indian Dermatol Online J. 2012;3:114-8.

43. Guadarrama-Bahena D, Arroyo-Yllanes ME, Magaña-García M, Madrigal-Pichardo BI. Prevalencia de alteraciones oftalmológicas en pacientes pediátricos con vitíligo. Rev Mex Oftalmol. 2003;77:6-9.

44. Tosti A, Bardazzi F, Tosti G, Monti L. Audiologic abnormalities in cases of vitiligo. J Am Acad Dermatol. 1987;17:230-3.

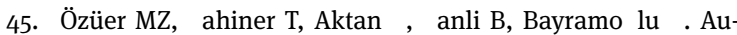
ditory evoked potentials in vitiligo patients. Scand Audiol. 1998;27:255-8.

46. Al-Mutairi N, Al-Sebeih K. Late onset vitiligo and audiological abnormalities: Is there any association? Indian J Dermatol Venereol Leprol. 2011;77:571-6.

47. Orecchia G, Marelli MA, Fresa D, Robiolio L. Audiologic disturbances in vitiligo. J Am Acad Dermatol. 1989;21:1317-8.

48. Hong $\mathrm{CK}$, Lee $\mathrm{MH}$, Jeong $\mathrm{KH}$, Cha CI, Yeo SG. Clinical analysis of hearing levels in vitiligo patients. Eur J Dermatol. 2009;19:50-6.

49. Aslan S, Serarslan G, Teksoz E, Dagli S. Audiological and transient evoked otoacoustic emission findings in patients with vitiligo. Otolaryngol Head Neck Surg. 2010;142:409-14.

50. Yamaguchi Y, Hearing VJ. Melanocytes and their diseases. Cold Spring Harb Perspect Med. 2014;4 a017046.

51. Huggins RH, Janusz CA, Schwartz RA. Vitiligo: A sign of systemic disease. Indian J Dermatol Venereol Leprol. 2006;72:68-71.

52. Yu H-S. Melanocyte destruction and repigmentation in vitiligo: A model for nerve cell damage and regrowth. J Biomed Sci. 2002;9:564-73.

53. Akay B, Bozkir M, Anadolu Y, Gullu S. Epidemiology of vitiligo, associated autoimmune diseases and audiological abnormalities: Ankara study of 80 patients in Turkey: Clinical, laboratory and genetic aspects of vitiligo. J Eur Acad Dermatol Venereol. 2010;24:1144-50.

54. Que SKT, Weston G, Suchecki J, Ricketts J. Pigmentary disorders of the eyes and skin. Clin Dermatol. 2015;33:147-58. 